\title{
The core of a class of non-atomic games which arise in economic applications*
}

\author{
Ezra Einy $^{1}$, Diego Moreno $^{2}$, Benyamin Shitovitz ${ }^{3}$ \\ ${ }^{1}$ Department of Economics, Ben-Gurion University of the Negev, Beer Sheva 84105, Israel \\ (e-mail: einy@bgumail.bgu.ac.il) \\ ${ }^{2}$ Departmento de Economia, Universidad Carlos III de Madrid, E-28903 Getafe, Spain \\ (e-mail: dmoreno@eco.uc3m.es) \\ ${ }^{3}$ Department of Economics, University of Haifa, Mount Carmel, Haifa 31905, Israel \\ (e-mail: binya@econ.haifa.ac.il)
}

Received May 1998/Revised version September 1998

\begin{abstract}
We study the core of a non-atomic game $v$ which is uniformly continuous with respect to the DNA-topology and continuous at the grand coalition. Such a game has a unique DNA-continuous extension $\bar{v}$ on the space $\boldsymbol{B}_{1}$ of ideal sets. We show that if the extension $\bar{v}$ is concave then the core of the game $v$ is non-empty iff $\bar{v}$ is homogeneous of degree one along the diagonal of $\boldsymbol{B}_{1}$. We use this result to obtain representation theorems for the core of a nonatomic game of the form $v=f \circ \mu$ where $\mu$ is a finite dimensional vector of measures and $f$ is a concave function. We also apply our results to some nonatomic games which occur in economic applications.
\end{abstract}

Key words: Coalitional game, core, non-atomic games

\section{Introduction}

One of the fundamental game theoretic concepts is the core of a coalitional game. It is the set of all feasible outcomes that no player or group of participants can improve upon by acting for themselves. The core of coalitional games with a finite or infinite set of players was investigated in many works (for a comprehensive survey see Kannai (1992)). In this work we consider non-atomic games which are uniformly continuous with respect to the discrete $N A$-topology and continuous at the grand coalition. Any such game has a

* We are indebted to Abraham Neyman for very helpful discussions concerning this work. Part of this work was done while Einy and Shitovitz visited the Department of Economics of Universidad Carlos III de Madrid. The support of the Department is gratefully acknowledged. Moreno acknowledges the support of the Spanish Ministry of Education (DGICYT), grant PB96-0118. 
unique extension which is continuous with respect to the discrete $N A$-topology on the space of ideal sets. We study the core of the class of such games whose extension is concave on the space of ideal sets. This class includes, for example, every game of the form $v=f \circ \mu$ where $\mu$ is a finite dimensional vector of non-atomic measures on a measurable space $(T, \Sigma)$ and $f$ is a continuous function at $\mu(T)$ and concave on the range of $\mu$. Such games occur in several economic applications. For example, every non-atomic glove market game and every non-atomic linear production game of Billera and Raanan (1981) are of this form and so is any Aumann-Shapley-Shubik market game of an atomless economy with a finite number of types (see Section 5). Large production games with a production function which satisfies decreasing returns to scale are also in this class (they have the form $f \circ \mu$ where $\mu$ represents the distribution of production factors among the owners and $f$ is the production function).

We prove that a game in the above-mentioned class has a non-empty core iff its (unique) extension to the space of ideal sets is homogeneous of degree one along the diagonal of the space $\boldsymbol{B}_{1}$ of ideal sets (see Theorem A). The game is totally balanced (i.e., every subgame has a non-empty core) iff its extension is homogeneous of degree one on all $\boldsymbol{B}_{1}$ (see Theorem B). As a consequence we obtain that such a game is balanced iff it is superadditive with respect to the grand coalition and it is totally balanced iff it is superadditive (see Corollaries 3.4 and 3.5). We also prove representation theorems for the core of a game of the form $v=f \circ \mu$ where $\mu$ is a finite dimensional vector of non-atomic measures and $f$ is a concave function on the range of $\mu$. The theorems are stated in terms of the supergradients of the function $f$ (see Theorems $\mathrm{C}$ and $\mathrm{D}$ ).

In the last section of the paper (see Section 5) we apply our main results to some non-atomic games which occur in economic applications.

\section{Preliminaries}

In this section we define some basic notions which are relevant to our work and prove some preliminary results which we use in the sequel.

Let $(T, \Sigma)$ be a measurable space, i.e., $T$ is a set and $\Sigma$ is a $\sigma$-field of subsets of $T$. We refer to the members of $T$ as players and to those of $\Sigma$ as coalitions. A coalitional game, or simply a game on $(T, \Sigma)$, is a function $v: \Sigma \rightarrow \mathfrak{R}_{+}$with $v(\varnothing)=0$. A game $v$ on $(T, \Sigma)$ is continuous at $S \in \Sigma$ if for all sequences $\left\{S_{n}\right\}_{n 11}^{\infty}$ of coalitions such that $S_{n+1} \supset S_{n}$ and $\bigcup_{n 1}^{\infty} S_{n}=S$, and all sequences $\left\{S_{n}\right\}_{n 1}^{\infty}$ of coalitions such that $S_{n+1} \subset S_{n}$ and $\bigcap_{n}^{\infty} S_{n}=S$, we have $v\left(S_{n}\right) \rightarrow v(S)$.

A payoff measure in a game $v$ is a bounded finitely additive measure $\lambda: \Sigma \rightarrow \mathfrak{R}$ which satisfies $\lambda(T) \leq v(T)$. The core of a game $v$, denoted by Core $(v)$, is the set of all payoff measures $\lambda$ such that $\lambda(S) \geq v(S)$ for all $S \in \Sigma$. As observed by Schmeidler (see the first part of the proof of Theorem 3.2 in Schmeilder (1972)), if $v$ is a continuous game at $T$, then every member of Core $(v)$ is countably additive.

The Banach space of all bounded Borel measurable functions with the supremum norm will be denoted by $\boldsymbol{B}=\boldsymbol{B}(T, \Sigma)$ and by $b a=b a(T, \Sigma)$, we denote the Banach space of all bounded finitely additive measures on $(T, \Sigma)$ 
with the variation norm. It is well known that $b a$ is the norm dual of $\boldsymbol{B}$. The subspace of $b a$ which consists of all bounded countably additive non-atomic measures on $(T, \Sigma)$ is denoted by $N A=N A(T, \Sigma)$. The $N A$-topology on $\boldsymbol{B}$ is the weakest topology such that for each $\lambda \in N A$ the function $f \rightarrow \int_{T} f d \lambda$ is continuous on $\boldsymbol{B}$. The DNA-topology on $\boldsymbol{B}$ (see Mertens (1980)) is defined in the same way, but when the real line $\mathfrak{R}$ is taken with the discrete topology. Note that a basis for the neighborhoods of $f \in \boldsymbol{B}$ in the $D N A$-topology consists of sets of the form $\{g \in \boldsymbol{B} \mid \mu(g)=\mu(f)\}$, where $\mu$ is a finite dimensional vector of measures in $N A$ (if $\mu=\left(\mu_{1}, \ldots, \mu_{m}\right)$ is a vector of measures in $N A$ and $f \in \boldsymbol{B}$ then $\mu(f)$ stands for $\left.\left(\int_{T} f d \mu_{1}, \ldots, \int_{T} f d \mu_{m}\right)\right)$. In the rest of the paper we denote by $\boldsymbol{B}_{1}=\boldsymbol{B}_{1}(T, \Sigma)$ the set of all functions $f$ in $\boldsymbol{B}$ such that $0 \leq f \leq 1$. If $v$ is a game on $(T, \Sigma)$ a function $w$ on $\boldsymbol{B}_{1}$ is an extension of $v$ if $w\left(1_{S}\right)=v(S)$ for every $S \in \Sigma$ (where here, and in the sequel, if $S \in \Sigma$ then $1_{S}$ denotes its indicator function). A game $v$ is DNA-uniformly continuous on $(T, \Sigma)$ if for every $\varepsilon>0$ there exists a finite dimensional vector $\mu$ of measures in NA such that for every $S_{1}$ and $S_{2}$ in $\Sigma$ we have

$$
\mu\left(S_{1}\right)=\mu\left(S_{2}\right) \Rightarrow\left|v\left(S_{1}\right)-v\left(S_{2}\right)\right|<\varepsilon .
$$

By Lyapunov's theorem, the indicator functions of the coalitions in $\Sigma$ are dense in $\boldsymbol{B}_{1}$ with respect to the DNA-topology (see also Corollary 22.3 in Aumann and Shapley (1974)). Therefore every DNA-uniformly continuous game $v$ has a unique extension $\bar{v}: \boldsymbol{B}_{1} \rightarrow \mathfrak{R}$ such that $\bar{v}$ is DNA-continuous on $\boldsymbol{B}_{1}$ (the proof is similar to that of Theorem G in Aumann and Shapley (1974)). It is clear that $\bar{v}$ is non-negative on $\boldsymbol{B}_{1}$.

We note that if $\bar{v}=g \circ \mu$ where $\mu$ is a finite dimensional vector of measures in NA and $g$ is a function defined on the range of $\mu$ then $\bar{v}(f)=g(\mu(f))$ for every $f \in \boldsymbol{B}_{1}$.

A coalition $S_{0} \in \Sigma$ is null in a game $v$ on $(T, \Sigma)$, if for every $S \in \Sigma$ such that $S \cap S_{0}=\varnothing$ we have $v\left(S \cup S_{0}\right)=v(S)$. Let $\mu$ be a countably additive measure in $b a_{+}$(where here, and in the sequel, if $A$ is a subset of an ordered vector space we denote by $A_{+}$the set of all non-negative members of $A$ ). A game $v$ is weakly absolutely continuous with respect to $\mu$ if every null set of $\mu$ is a null set of $v$.

Proposition 2.1. Let $v$ be DNA-uniformly continuous game on $(T, \Sigma)$. Then there exists a measure $\mu$ in $N A_{+}$such that $v$ is weakly absolutely continuous with respect to $\mu$.

Proof: Since $v$ is DNA-uniformly continuous on $(T, \Sigma)$, for every natural number $n$ there exists a vector $\mu^{n}=\left(\mu_{1}^{n}, \ldots, \mu_{m_{n}}^{n}\right)$ of measures in $N A_{+}$such that $\left\|\mu^{n}(T)\right\| \leq 1$ and for every $S_{1}, S_{2} \in \Sigma$

$$
\mu^{n}\left(S_{1}\right)=\mu^{n}\left(S_{2}\right) \Rightarrow\left|v\left(S_{1}\right)-v\left(S_{2}\right)\right|<\frac{1}{n} .
$$

For every $n$ let $\lambda_{n}=1 / m_{n} \sum_{i}^{m_{n}} \mu_{i}^{n}$ and let $\mu=\sum_{n}^{\infty}{ }^{\infty}{ }^{n} \lambda_{n}$. Then $\mu \in N A_{+}$. Now let $S_{0}$ be a null coalition of $\mu$. Then $\mu^{n}\left(S_{0}\right)=0$ for every $n$. Let $S \in \Sigma$ such that $S \cap S_{0}=\varnothing$. Then for every $n$ we have $\left|v\left(S \cup S_{0}\right)-v(S)\right|<1 / n$, and thus $S_{0}$ is a null coalition of $v$. Therefore $v$ is weakly absolutely continuous with respect to $\mu$.

Q.E.D. 
Proposition 2.2. Let $v$ be a DNA-uniformly continuous game on $(T, \Sigma)$ which is continuous at $T$. Then every member of the core of $v$ is in $N A_{+}$.

Proof: Let $\lambda \in \operatorname{Core}(v)$. Then it is clear that $\lambda$ is non-negative. As mentioned earlier, the continuity of $v$ at $T$ implies that $\lambda$ is countably additive. Now by Proposition 2.1, there exists $\mu \in N A_{+}$such that $v$ is weakly absolutely continuous with respect to $\mu$. We show that $\lambda$ is absolutely continuous with respect to $\mu$. Indeed, let $S \in \Sigma$ such that $\mu(S)=0$. As $\lambda \in \operatorname{Core}(v)$ we have

$$
0 \leq \lambda(S)=\lambda(T)-\lambda(T \backslash S) \leq v(T)-v(T \backslash S)=0
$$

Hence, $\lambda(S)=0$. Therefore $\lambda$ is absolutely continuous with respect to $\mu$. We will show that $\lambda \in N A_{+}$. Assume, on the contrary, that $A$ is an atom of $\lambda$. Then $\lambda(A)>0$. Let $0<\varepsilon<\lambda(A)$. Since $\lambda$ is absolutely continuous with respect to $\mu$, there exists $\delta>0$ such that for every $S \in \Sigma$

$$
\mu(S)<\delta \Rightarrow \lambda(S)<\varepsilon .
$$

Let $n$ be a natural number such that $n \delta>\mu(A)$. Since $\mu \in N A_{+}$, there exists a partition $A_{1}, \ldots, A_{n}$ of $A$ such that for every $1 \leq i \leq n$ we have $A_{i} \in \Sigma$ and $\mu\left(A_{i}\right)=(1 / n) \mu(A)<\delta$. As $A$ is an atom of $\lambda$, there exists $1 \leq i \leq n$ such that $\lambda(A)=\lambda\left(A_{i}\right)<\varepsilon$, which is a contradiction.

Q.E.D.

\section{Existence of the core}

In this section we investigate the existence of the core of a game $v$ which is DNA-uniformly continuous on $(T, \Sigma)$ and its extension $\bar{v}$ to $\boldsymbol{B}_{1}$ is concave.

We start with a theorem which gives a necessary and sufficient condition for non-emptiness of the core of such a game.

Theorem A. Let $v$ be a DNA-uniformly continuous game on $(T, \Sigma)$ which is continuous at $T$. Assume that its extension $\bar{v}$ is concave on $\boldsymbol{B}_{1}$. Then the core of the game $v$ is non-empty iff $\bar{v}$ is homogeneous of degree one along the diagonal of $\boldsymbol{B}_{1}$. That is, $\bar{v}\left(\alpha 1_{T}\right)=\alpha \bar{v}\left(1_{T}\right)$ for every $0 \leq \alpha \leq 1$.

For the proof of Theorem A we need some preparation. We start with the following proposition which is interesting on its own.

Proposition 3.1. Let $v$ be a DNA-uniformly continuous game which is continuous at $T$. Assume that Core $(v) \neq \varnothing$. Then for every $0 \leq \alpha \leq 1$ we have

$$
\bar{v}\left(\alpha 1_{T}\right) \leq \alpha \bar{v}\left(1_{T}\right)
$$

Proof: Assume, on the contrary, that there exists $0<\alpha<1$ such that $\bar{v}\left(\alpha 1_{T}\right)>\alpha \bar{v}\left(1_{T}\right)$. Let $\varepsilon=\bar{v}\left(\alpha 1_{T}\right)-\alpha v(T)$ and let $\lambda \in \operatorname{Core}(v)$. Then by Proposition 2.2, $\lambda \in N A_{+}$. Since $\bar{v}$ is DNA continuous at $\alpha 1_{T}$, there exists a finite dimensional vector $\mu$ of measures in $N A$ such that for every $f \in \boldsymbol{B}_{1}$

$$
\mu(f)=\alpha \mu(T) \Rightarrow\left|\bar{v}(f)-\bar{v}\left(\alpha 1_{T}\right)\right|<\varepsilon
$$


Now by Lyapunov's theorem, there exists $S \in \Sigma$ such that $\mu(S)=\alpha \mu(T)$ and $\lambda(S)=\alpha \lambda(T)$. Since $\lambda \in \operatorname{Core}(v)$, we have

$$
\lambda(S) \geq v(S)>\bar{v}\left(\alpha 1_{T}\right)-\varepsilon=\alpha v(T)=\alpha \lambda(T),
$$

which is a contradiction.

Q.E.D.

Let $F$ be a real valued function defined on a subset $K$ of a topological vector space $X$. A liner functional $\psi$ on $X$ is a supergradient of $F$ at a point $x_{0} \in K$ if for every $x \in K$ we have

$$
F(x) \leq F\left(x_{0}\right)+\psi\left(x-x_{0}\right)
$$

The set of all supergradients of $F$ at $x_{0}$ is denoted by $\partial F\left(x_{0}\right)$. It is well known that if $K$ is a convex set and $F$ is a concave function on $K$ and $x_{0}$ is a point in the relative interior of $K\left(x_{0}\right.$ is in the relative interior of $K$ if for every $x \in K \backslash\left\{x_{0}\right\}$ there exist $y \in K$ and $0<\alpha<1$ such that $x_{0}=\alpha x+(1-\alpha) y$ ) then $\partial F\left(x_{0}\right) \neq \varnothing$ (see, for example, the theorem on p. 23 of Holmes (1975)).

Proof of Theorem A: Assume that $\operatorname{Core}(v) \neq \varnothing$. Then since $\bar{v}$ is concave, Proposition 3.1 implies that $\bar{v}\left(\alpha 1_{T}\right)=\alpha \bar{v}\left(1_{T}\right)$ for every $0 \leq \alpha \leq 1$.

We now show that if $\bar{v}$ is homogeneous of degree one along the diagonal of $\boldsymbol{B}_{1}$, then $\operatorname{Core}(v) \neq \varnothing$.

For every natural number $n>1$ we have $(1-1 / n) 1_{T} \in \operatorname{int} \boldsymbol{B}_{1}$ and thus, $\partial \bar{v}\left((1-1 / n) 1_{T}\right) \neq \varnothing$. For every $n$ let $\psi_{n} \in \partial \bar{v}\left((1-1 / n) 1_{T}\right)$. Then

$$
\begin{aligned}
0 & =\bar{v}(0) \leq \bar{v}\left(\left(1-\frac{1}{n}\right) 1_{T}\right)-\left(1-\frac{1}{n}\right) \psi_{n}\left(1_{T}\right) \\
& =\left(1-\frac{1}{n}\right) v(T)-\left(1-\frac{1}{n}\right) \psi_{n}\left(1_{T}\right)
\end{aligned}
$$

Therefore $\psi_{n}\left(1_{T}\right) \leq v(T)$. On the other hand,

$$
v(T) \leq \bar{v}\left(\left(1-\frac{1}{n}\right) 1_{T}\right)+\frac{1}{n} \psi_{n}\left(1_{T}\right)=\left(1-\frac{1}{n}\right) v(T)+\frac{1}{n} \psi_{n}\left(1_{T}\right) .
$$

Hence, $\psi_{n}\left(1_{T}\right) \geq v(T)$ and thus $\psi_{n}\left(1_{T}\right)=v(T)$.

Now for every $g \in \boldsymbol{B}_{1}$ we have

$$
\bar{v}(g) \leq \bar{v}\left(\left(1-\frac{1}{n}\right) 1_{T}\right)+\psi_{n}(g)-\left(1-\frac{1}{n}\right) \psi_{n}\left(1_{T}\right)=\psi_{n}(g) .
$$

As $\bar{v} \geq 0, \psi_{n}$ is a positive linear functional on $\boldsymbol{B}$ (i.e., $\psi_{n}(g) \geq 0$ for every $\left.g \in \boldsymbol{B}_{+}\right)$. Therefore for every $n$

$$
\left\|\psi_{n}\right\|=\psi_{n}\left(1_{T}\right)=v(T) .
$$

Thus, the sequence $\left(\psi_{n}\right)_{n 2}^{\infty}$ is a uniformly bounded sequence of continuous linear functionals on $\boldsymbol{B}$. Let $\psi$ be a weak*-cluster point of the sequence 
$\left(\psi_{n}\right)_{n 2}^{\infty}$. Then $\psi\left(1_{T}\right)=v(T)$. We show that $\psi \in \partial \bar{v}\left(1_{T}\right)$. Assume, on the contrary, that $\psi \notin \partial \bar{v}\left(1_{T}\right)$. Then there exists $f \in \boldsymbol{B}_{1}$ such that

$$
\bar{v}(f)>v(T)+\psi(f)-\psi\left(1_{T}\right)=\psi(f)
$$

Let $\varepsilon=\bar{v}(f)-\psi(f)$. Then the set $U=\left\{\varphi \in \boldsymbol{B}^{*}|| \varphi(f)-\psi(f) \mid<\varepsilon\right\}$ is a weak*-neighborhood of $\psi$ in $\boldsymbol{B}^{*}$. Therefore there exists $n$ such that $\psi_{n} \in U$. Hence, $\psi_{n}(f)<\psi(f)+\varepsilon=\bar{v}(f)$, which contradicts (3.1). For every $S \in \Sigma$ let $\lambda(S)=\psi\left(1_{S}\right)$. Then $\lambda \in b a_{+}$and $\lambda(T)=v(T)$. Since $\psi \in \partial \bar{v}\left(1_{T}\right)$, for every $S \in \Sigma$ we have

$$
v(S) \leq v(T)+\psi\left(1_{S}\right)-\psi\left(1_{T}\right)=\lambda(S) .
$$

Thus, $\lambda \in \operatorname{Core}(v)$.

Q.E.D.

If $\mu$ is a finite dimensional vector of measures in $N A$ we denote by $R(\mu)$ the range of $\mu$.

The following corollary is an immediate consequence of Theorem A.

Corollary 3.2. Let $\mu$ be a finite dimensional vector of measures in $N A$ and let $f: R(\mu) \rightarrow \mathfrak{R}_{+}$be a concave function which is continuous at $\mu(T)$ and satisfies $f(0)=0$. Then the core of the game $v=f \circ \mu$ is non-empty iff for every $0 \leq \alpha \leq 1$ we have $f(\alpha \mu(T))=\alpha f(\mu(T))$.

Let $S \in \Sigma$. Denote $\Sigma_{S}=\{Q \in \Sigma \mid Q \subset S\}$. Then $\Sigma_{S}$ is a $\sigma$-field of subsets of $S$. Let $v$ be a game on $(T, \Sigma)$, and let $S \in \Sigma$. The subgame of $v$ which is determined by $S$ is the game $v_{S}$ on $\left(S, \Sigma_{S}\right)$ which is given by $v_{S}(Q)=v(Q)$ for every $Q \in \Sigma_{S}$. A game $v$ on $(T, \Sigma)$ is called totally balanced if for every $S \in \Sigma$ we have $\operatorname{Core}\left(v_{S}\right) \neq \varnothing$.

Theorem B. Let $v$ be a DNA-uniformly continuous game. Assume that $v$ is continuous at every coalition in $\Sigma$ and that $\bar{v}$ is concave on $\boldsymbol{B}_{1}$. Then $v$ is totally balanced iff $\bar{v}$ is homogeneous of degree one on $\boldsymbol{B}_{1}$. That is, for every $0 \leq \alpha \leq 1$ and $f \in \boldsymbol{B}_{1}$ we have $\bar{v}(\alpha f)=\alpha \bar{v}(f)$.

Proof: Assume that $v$ is totally balanced. Let $S \in \Sigma$. Since Core $\left(v_{S}\right) \neq \varnothing$ and $v_{S}$ is continuous at $S$, by applying Theorem A to the game $v_{S}$ on $\left(S, \Sigma_{S}\right)$, we obtain that $\bar{v}\left(\alpha 1_{S}\right)=\bar{v}_{S}\left(\alpha 1_{S}\right)=\alpha \bar{v}_{S}\left(1_{S}\right)=\alpha \bar{v}\left(1_{S}\right)$ for every $0 \leq \alpha \leq 1$. Since the indicator functions are DNA-dense in $\boldsymbol{B}_{1}$, we obtain that $\bar{v}(\alpha f)=\alpha \bar{v}(f)$ for every $f \in \boldsymbol{B}_{1}$ and $0 \leq \alpha \leq 1$.

Assume now that $\bar{v}$ is homogeneous of degree one on $\boldsymbol{B}_{1}$. Let $S \in \Sigma$. Then $\bar{v}\left(\alpha 1_{S}\right)=\alpha \bar{v}(S)$ for every $0 \leq \alpha \leq 1$. As $v$ is continuous at every coalition in $\Sigma$, we can apply Theorem A to the game $v_{S}$ on the space $\left(S, \Sigma_{S}\right)$ and deduce that $\operatorname{Core}\left(v_{S}\right) \neq \varnothing$.

Q.E.D.

Corollary 3.3. Let $\mu$ be a finite dimensional vector of measures in NA. Assume that $f: R(\mu) \rightarrow \mathfrak{R}_{+}$is a continuous concave function which satisfies $f(0)=0$. Then the game $v=f \circ \mu$ is totally balanced iff $f$ is homogeneous of degree one on $R(\mu)$ (i.e., $f(\alpha x)=\alpha f(x)$ for every $x \in R(\mu)$ and $0 \leq \alpha \leq 1$ ). 
In light of Corollaries 3.2 and 3.3 it will be useful to give an example of a function $f$ which is defined on the range $R$ of a vector of non-atomic measures on a measurable space and such that $f$ is continuous, non-decreasing and concave on $R, f(0)=0, f$ is homogeneous of degree one along the diagonal of $R$, but $f$ is not homogeneous of degree one in the entire range $R$. Indeed, let $R$ be the unit square in $\mathfrak{R}^{2}\left(R\right.$ is, for example, the range of the vector $\left(\lambda_{1}, \lambda_{2}\right)$ when the measureable space is $[0,2]$ with its Borel subsets, $\lambda_{1}$ is the Lebesgue measure on $[0,1]$ and $\lambda_{2}$ is the Lebesgue measure on $\left.[1,2]\right)$. Define a function $f$ on $R$ by

$$
f(x, y)=\sqrt{ } x y\left(1-\varepsilon(x-y)^{2}\right),
$$

where $0<\varepsilon<10^{7}$. It is clear that $f$ is continuous on $R$ and homogeneous of degree one along the diagonal of $R$ but not in all $R$. It is also easy to check (by computing the partial derivatives) that $f$ is non-decreasing. A direct computation gives that the Hessian of $f$ is negative semidefinite on $R$. Therefore $f$ is concave on $R$.

A game $v$ on $(T, \Sigma)$ is superadditive with respect to a coalition $S \in \Sigma$ if for every two disjoint coalitions $S_{1}, S_{2} \in \Sigma$ such that $S_{1} \cup S_{2}=S$ we have $v(S) \geq v\left(S_{1}\right)+v\left(S_{2}\right)$. A game $v$ is superadditive if it is superadditive with respect to any coalition in $\Sigma$.

The following two corollaries are consequences of Theorems A and B respectively.

Corollary 3.4. Let $v$ be a DNA-uniformly continuous game on $(T, \Sigma)$ which is continuous at $T$. Assume that its extension $\bar{v}$ is concave on $\boldsymbol{B}_{1}$. Then the core of the game $v$ is non-empty iff $v$ is superadditive with respect to the grand coalition.

Proof: It is easy to see that if $\operatorname{Core}(v) \neq \varnothing$ then $v$ is superadditive with respect to the grand coalition. Assume that $v$ is superadditive with respect to the grand coalition. We will show that $\operatorname{Core}(v) \neq \varnothing$. Let $0 \leq \alpha \leq 1$. By theorem $\mathrm{A}$, it is sufficient to show that $\bar{v}\left(\alpha 1_{T}\right)=\alpha \bar{v}\left(1_{T}\right)$. Since $\bar{v}$ is concave on $\boldsymbol{B}_{1}$, we have $\bar{v}\left(\alpha 1_{T}\right) \geq \alpha \bar{v}\left(1_{T}\right)$. So it remains to show that $\bar{v}\left(\alpha 1_{T}\right) \leq \alpha \bar{v}\left(1_{T}\right)$. We first show that

$$
\bar{v}\left(1_{T}\right) \geq \bar{v}\left(\alpha 1_{T}\right)+\bar{v}\left((1-\alpha) 1_{T}\right) .
$$

Assume, on the contrary, that (3.2) is not satisfied. Let $\varepsilon=\bar{v}\left(\alpha 1_{T}\right)+$ $\bar{v}\left((1-\alpha) 1_{T}\right)-\bar{v}\left(1_{T}\right)$. Then $\varepsilon>0$. Since $\bar{v}$ is continuous at $\alpha 1_{T}$ and $(1-\alpha) 1_{T}$, there exist two finite dimensional vectors of measures $\mu^{1}$ and $\mu^{2}$ in NA such that for every $f \in \boldsymbol{B}_{1}$

$$
\mu^{1}(f)=\alpha \mu^{1}(T) \Rightarrow\left|\bar{v}(f)-\bar{v}\left(\alpha 1_{T}\right)\right|<\frac{\varepsilon}{2}
$$

and

$$
\mu^{2}(f)=(1-\alpha) \mu^{2}(T) \Rightarrow\left|\bar{v}(f)-\bar{v}\left((1-\alpha) 1_{T}\right)\right|<\frac{\varepsilon}{2} .
$$

Let $\mu=\left(\mu^{1}, \mu^{2}\right)$. Then there exists $S \in \Sigma$ such that $\mu(S)=\alpha \mu(T)$. There- 
fore $\mu(T \backslash S)=(1-\alpha) \mu(T)$. Hence,

$$
\left|v(S)-\bar{v}\left(\alpha 1_{T}\right)\right|<\frac{\varepsilon}{2}
$$

and

$$
\left|v(T \backslash S)-\bar{v}\left((1-\alpha) 1_{T}\right)\right|<\frac{\varepsilon}{2} .
$$

Now (3.3) and (3.4) imply that $v\left(\alpha 1_{T}\right)+v\left((1-\alpha) 1_{T}\right)-(v(S)+v(T \backslash S))<\varepsilon$. This yields that

$$
v(T)<v(S)+v(T \backslash S),
$$

which contradicts the assumption that $v$ is superadditive with respect to the grand coalition. Therefore

$$
\bar{v}\left(1_{T}\right) \geq \bar{v}\left(\alpha 1_{T}\right)+\bar{v}\left((1-\alpha) 1_{T}\right) .
$$

This inequality and the concavity of $\bar{v}$ imply that

$$
\bar{v}\left(\alpha 1_{T}\right) \leq \bar{v}\left(1_{T}\right)-(1-\alpha) \bar{v}\left(1_{T}\right)=\alpha \bar{v}\left(1_{T}\right) .
$$

Corollary 3.5. Let $v$ be a DNA-uniformly continuous game. Assume that $v$ is continuous at every coalition in $\Sigma$ and that $\bar{v}$ is concave on $\boldsymbol{B}_{1}$. Then $v$ is totally balanced iff it is superadditive.

Proof: It is clear that if $v$ is totally balanced then it is superadditive. Assume that $v$ is superadditive. We show that it is totally balanced. Let $0 \leq \alpha \leq 1$. By Theorem $\mathrm{B}$, it is sufficient to show that for every $f \in \boldsymbol{B}_{1}$ we have $\bar{v}(\alpha f)=$ $\alpha \bar{v}(f)$. Let $f \in \boldsymbol{B}_{1}$. Since $\bar{v}$ is concave, $\bar{v}(\alpha f) \geq \alpha \bar{v}(f)$. Note now that if $\mu$ is a finite dimensional vector of measures in NA, then by Theorem 4 in Dvoretsky, Wald and Wolfowitz (1951) there exists disjoint coalitions $S_{1}$, $S_{2} \in \Sigma$ such that $\mu\left(S_{1}\right)=\alpha \mu(f)$ and $\mu\left(S_{2}\right)=(1-\alpha) \mu(f)$. Therefore we can apply an argument similar to that in the proof of Corollary 3.4 to obtain that

$$
\bar{v}(f) \geq \bar{v}(\alpha f)+\bar{v}((1-\alpha) f),
$$

which implies that $\bar{v}(\alpha f) \leq \alpha \bar{v}(f)$.

Q.E.D.

\section{Representation theorems for the core}

In this section we state and prove some representation theorems for the core of a subclass of the class of games which were studied in Section 3. We start with the following theorem.

Theorem C. Let $\mu$ be a finite dimensional vector of measures in NA. Assume that $f: R(\mu) \rightarrow \mathfrak{R}_{+}$is a concave function which is continuous at $\mu(T)$ and 
satisfies $f(0)=0$. Then the core of the game $v=f \circ \mu$ is given by

$$
\text { Core }(v)=\{p \cdot \mu \mid p \in \partial f(\mu(T)) \text { and } p \cdot \mu(T)=f(\mu(T))\}
$$

Proof: Let $M(v)=\{p \cdot \mu \mid p \in \partial f(\mu(T))$ and $p \cdot \mu(T)=f(\mu(T))\}$. We first show that $M(v) \subset \operatorname{Core}(v)$. Indeed, let $\lambda \in M(v)$. Then there exists $p \in$ $\partial f(\mu(T))$ such that $p \cdot \mu(T)=f(\mu(T))$ and $\lambda=p \cdot \mu$. For every $S \in \Sigma$ we have

$$
\lambda(S)=\lambda(T)-\lambda(T \backslash S)=f(\mu(T))-p \cdot \mu(T \backslash S) \geq f(\mu(S))=v(S)
$$

Therefore $\lambda \in \operatorname{Core}(v)$.

We now show that Core $(v) \subset M(v)$. Without loss of generality, Core $(v) \neq$ $\varnothing$. Then by Corollary 3.2, we have $f(\alpha \mu(T))=\alpha f(\mu(T))$ for every $0 \leq \alpha \leq$ 1. Now by Corollary 2.6 in Billera and Raanan (1981) and the remark that follows it, every member of Core $(v)$ is a linear combination of the components of $\mu$. Let $\lambda \in \operatorname{Core}(v)$. Then there exists a vector $p$ such that $\lambda=p \cdot \mu$. Now for every $S \in \Sigma$ we have

$$
f(\mu(S)) \leq \lambda(S)=p \cdot \mu(S)+f(\mu(T))-p \cdot \mu(T) .
$$

Thus, $p \in \partial f(\mu(T))$ and $\lambda \in M(v)$.

Q.E.D.

Usually in economic applications (see the examples in Section 5) the function $f$ of Theorem $\mathrm{C}$ is the restriction of a non-decreasing concave function $\bar{f}$ which is defined on all $\mathfrak{R}_{+}^{m}$. Moreover, when we have a non-decreasing concave function $f$ which is defined on the range of a vector of measures $\mu=\left(\mu_{1}, \ldots, \mu_{m}\right)$ in $N A_{+}$it is more convenient to compute the supergradients at $\mu(T)$ of a non-decreasing concave extension $\bar{f}$ of $f$ to $\mathfrak{R}_{+}^{m}$. Such an extension always exists when $f$ is concave and non-decreasing on the range $R(\mu)$ of $\mu$. For example, the function $\bar{f}: \mathfrak{R}_{+}^{m} \rightarrow \mathfrak{R}$ which is given by

$$
\bar{f}(x)=\max \{f(y) \mid y \in R(\mu), y \leq x\}
$$

is such an extension.

We can now state and prove the main result of this section.

Theorem D. Let $\mu=\left(\mu_{1}, \ldots, \mu_{m}\right)$ be a vector of non-trivial measures in $N A_{+}$. Assume that $f: R(\mu) \rightarrow \mathfrak{R}$ is a non-decreasing concave function which is continuous at $\mu(T)$ and satisfies $f(0)=0$. Let $\bar{f}: \mathfrak{R}_{+}^{m} \rightarrow \mathfrak{R}$ be a non-decreasing concave extension of $f$. Then the core of the game $v=f \circ \mu$ is given by

$$
\operatorname{Core}(v)=\{p \cdot \mu \mid p \in \partial \bar{f}(\mu(T)) \text { and } p \cdot \mu(T)=f(\mu(T))\}
$$

Proof: Let $\bar{M}(v)=\{p \cdot \mu \mid p \in \partial \bar{f}(\mu(T))$ and $p \cdot \mu(T)=f(\mu(T))\}$.

Since $\partial \bar{f}(\mu(T)) \subset \partial f(\mu(T))$, Theorem $\mathrm{C}$ implies that $\bar{M}(v) \subset$ Core $(v)$. We will show that $\operatorname{Core}(v) \subset \bar{M}(v)$. By Theorem $\mathrm{C}$, it is sufficient to show that for every $p \in \partial f(\mu(T))$ with $p \cdot \mu(T)=f(\mu(T))$ there exists $q \in \partial \bar{f}(\mu(T))$ 
such that $p \cdot \mu=q \cdot \mu$. Let $p \in \partial f(\mu(T))$ be such that $p \cdot \mu(T)=f(\mu(T))$. We first show that for every $x \in R(\mu)$ there exists $q \in \partial \bar{f}(\mu(T))$ such that $p \cdot x \leq q \cdot x$. Let $x \in R(\mu)$. Then $x=\mu(S)$ for some $S \in \Sigma$. For every natural number $n>1$ let $S_{n} \in \Sigma$ be such that $\mu\left(S_{n}\right)=(1 / n) \mu(S)$ and $p \cdot \mu\left(S_{n}\right)=$ $(1 / n) p \cdot \mu(S)$. For every $n$ let $q_{n} \in \partial \bar{f}\left(\mu\left(T \backslash S_{n}\right)\right)$. Since $\bar{f}$ is non-decreasing on $\mathfrak{R}_{+}^{m}, q_{n} \geq 0$ for every $n$. As $p \cdot \mu \in \operatorname{Core}(v)$, for every $n$ we have

$$
\begin{aligned}
0=f(0) & \leq f\left(\mu\left(T \backslash S_{n}\right)\right)-q_{n} \cdot \mu(T)+\frac{1}{n} q_{n} \cdot \mu(S) \\
& \leq p \cdot \mu(T)-p \cdot \mu\left(S_{n}\right)-q_{n} \cdot \mu(T)+\frac{1}{n} q_{n} \cdot \mu(T) .
\end{aligned}
$$

Therefore

$$
\left(1-\frac{1}{n}\right) q_{n} \cdot \mu(T) \leq f(\mu(T)) .
$$

Since $\mu_{i}(T)>0$ for every $1 \leq i \leq m$, and $q_{n} \geq 0$ for every $n$, the sequence $\left(q_{n}\right)_{n 2}^{\infty} 2$ is bounded, and therefore it has a convergent subsequence which converges to a vector $q \in \mathfrak{R}_{+}^{m}$. It is clear that $q \in \partial \bar{f}(\mu(T))$. We will show that $p \cdot x \leq q \cdot x$. Indeed, for every $n$ we have

$$
f(\mu(T)) \leq f\left(\mu\left(T \backslash S_{n}\right)\right)+q_{n} \cdot \mu\left(S_{n}\right) \leq p \cdot \mu\left(T \backslash S_{n}\right)+q_{n} \cdot \mu\left(S_{n}\right) .
$$

As $p \cdot \mu(T)=f(\mu(T))$, we obtain

$$
\frac{1}{n} p \cdot x \leq \frac{1}{n} q_{n} \cdot x .
$$

Thus, $p \cdot x \leq q_{n} \cdot x$ for every $n$. Therefore $p \cdot x \leq q \cdot x$. by

Define now a function $H: \partial \bar{f}(\mu(T)) \times R(\mu) \rightarrow \mathfrak{R}$

$$
H(q, x)=q \cdot x-p \cdot x .
$$

Since $\partial \bar{f}(\mu(T))$ and $R(\mu)$ are convex and compact and $H$ is continuous and linear in each variable separately, by the minimax theorem

$$
\min _{x \in R(\mu)} \max _{q \in \partial \bar{f}(\mu(T))} H(q, x)=\max _{q \in \partial \bar{f}(\mu(T))} \min _{x \in R(\mu)} H(q, x) .
$$

We have just shown that for every $x \in R(\mu)$ there exists $q \in \partial \bar{f}(\mu(T))$ such that $H(q, x) \geq 0$. Therefore $\min _{x \in R(\mu)} \max _{q \in \partial \bar{f}(\mu(T))} H(q, x) \geq 0$. Hence by (4.1), there exists $q \in \partial \bar{f}(\mu(T))$ such that for every $x \in R(\mu)$ we have $H(q, x) \geq 0$. That is, $p \cdot x \leq q \cdot x$ for every $x \in R(\mu)$. Since $p \cdot \mu(T)=$ $f(\mu(T))$ and $q \cdot \mu(T) \leq f(\mu(T))$, we have $q \cdot \mu(T)=f(\mu(T))$. Now $p \cdot \mu$ and $q \cdot \mu$ are two measures on $(T, \Sigma)$ such that $p \cdot \mu(S) \leq q \cdot \mu(S)$ for every $S \in \Sigma$ and $p \cdot \mu(T)=q \cdot \mu(T)$. Therefore $p \cdot \mu=q \cdot \mu$ and the proof is complete.

Q.E.D. 
Remark 4.1: We note that Theorem D can be proved without using Theorems A and C by applying Sion's minimax theorem (e.g., Sion (1958)) to the function

$$
H: \partial \bar{f}(\mu(T)) \times \boldsymbol{B}_{1} \rightarrow \mathfrak{R} \quad \text { given by } H(p, g)=p \cdot \mu(g)-\lambda(g),
$$

where $\lambda$ is a fixed member of $\operatorname{Core}(v)$ and $\boldsymbol{B}_{1}$ is identified with the positive unit ball of $L_{\infty}(T, \Sigma, \sigma)$ where $\sigma=\sum_{i=1}^{m} \mu_{i}$ (and hence $\boldsymbol{B}_{1}$ is weak*-compact).

If $f$ is a function defined on a neighborhood of a point $x \in \mathfrak{R}_{+}^{m}$ and differentiable at $x$ we denote by $\nabla f(x)$ the gradient of $x$.

The following corollary is an immediate consequence of Theorem D.

Corollary 4.2. Let $\mu=\left(\mu_{1}, \ldots, \mu_{m}\right)$ be a vector of measures in $N A_{+}$. Assume that $f: \mathfrak{R}_{+}^{m} \rightarrow \mathfrak{R}$ is a non-decreasing concave function which is differentiable at $\mu(T)$ and satisfies $f(0)=0$. Then the core of the game $v=f \circ \mu$ is non-empty iff $\nabla f(\mu(T)) \cdot \mu(T)=f(\mu(T))$. Moreover, if Core $(v) \neq \varnothing$ then

$$
\operatorname{Core}(v)=\{\nabla f(\mu(T)) \cdot \mu\} .
$$

The following proposition deals with differentiable games which are not covered by Corollary 4.2.

Proposition 4.3. Let $\mu=\left(\mu_{1}, \ldots, \mu_{m}\right)$ be a vector of measures in $N A_{+}$and let $f: \mathfrak{R}_{+}^{m} \rightarrow \mathfrak{R}_{+}$be a differentiable function at $\mu(T)$ which satisfies $f(0)=0$. Assume that $\nabla f(\mu(T)) \in \partial f(\mu(T))$ and $\nabla f(\mu(T)) \cdot \mu(T)=f(\mu(T))$. Then the core of the game $v=f \circ \mu$ is given by

$$
\operatorname{Core}(v)=\{\nabla f(\mu(T)) \cdot \mu\} .
$$

Proof: Since $\nabla f(\mu(T)) \in \partial f(\mu(T))$, it is straightforward to check that $\nabla f(\mu(T)) \cdot \mu \in \operatorname{Core}(v)$. We will show that Core $(v) \subset\{\nabla f(\mu(T)) \cdot \mu\}$. Let $\lambda \in \operatorname{Core}(v)$ and $S \in \Sigma$. For every natural number $n$ let $S_{n} \in \Sigma$ such that $\lambda\left(S_{n}\right)=(1 / n) \lambda(S)$ and $\mu\left(S_{n}\right)=(1 / n) \mu(S)$. Since $\lambda \in \operatorname{Core}(v)$ and $f$ is differentiable at $\mu(T)$, we have

$$
\lambda\left(T \backslash S_{n}\right) \geq f\left(\mu\left(T \backslash S_{n}\right)\right)=f(\mu(T))-\frac{1}{n} \nabla f(\mu(T)) \cdot \mu(S)+o\left(\frac{1}{n}\right) .
$$

Therefore

$$
\lambda(S) \leq \nabla f(\mu(T)) \cdot \mu(S)+\alpha_{n},
$$

where $\lim _{n \rightarrow \infty} \alpha_{n}=0$.

Thus $\lambda(S) \leq \nabla f(\mu(T)) \cdot \mu(S)$ for every $S \in \Sigma$. As $\nabla f(\mu(T)) \cdot \mu(T)=$ $f(\mu(T))=\lambda(T)$, we must have $\lambda=\nabla f(\mu(T)) \cdot \mu$.

Q.E.D.

As an application of Proposition 4.3 we compute the core of the game in the following example.

Example 4.4: Consider the measurable space $([0,1], \beta)$ where $\beta$ is the $\sigma$-field of the Borel subsets of $[0,1]$ and let $\lambda$ be the Lebesgue measure on $([0,1], \beta)$. 
Define a function:

$$
f(x)= \begin{cases}3 x^{2} & 0 \leq x \leq \frac{1}{2} \\ -x^{2}+4 x-1 & x \geq \frac{1}{2}\end{cases}
$$

Then $f$ is not concave on $[0,1]$, but as $f^{\prime}(1) \in \partial f(1)$ and $f^{\prime}(1)=f(1)$, by Proposition 4.3, the core of the game $v=f \circ \lambda$ is $\{2 \lambda\}$.

\section{Applications}

In this section we apply Theorems $\mathrm{A}$ and $\mathrm{D}$ to games which arise in economic applications. We start with the non-atomic glove market game whose core was studied in Billera and Raanan (1981) and Einy et al. (1996).

Let $\mu_{1}, \ldots, \mu_{m}$ be non-atomic measures in $N A_{+}$. The non-atomic glove market game is defined by

$$
v(S)=\min \left(\mu_{1}(S), \ldots, \mu_{m}(S)\right) \text { for every } S \in \Sigma .
$$

Billera and Raanan (see Billera and Raanan (1981), Corollary 2.7) proved that the core of $v$ coincides with the convex hull of the set $M=\left\{\mu_{i} \mid i=\right.$ $1, \ldots, m$ and $\left.\mu_{i}(T)=v(T)\right\}$. We now derive this result from Theorem D. It is clear that $M \subset \operatorname{Core}(v)$. Since Core $(v)$ is convex, co $M \subseteq \operatorname{Core}(v)$ (co $M$ denotes the convex hull of $M)$. Define now $\bar{f}: \mathfrak{R}_{+}^{m} \rightarrow R$ by $\bar{f}\left(x_{1}, \ldots, x_{m}\right)=$ $\min \left(x_{1}, \ldots, x_{m}\right)$. Let $\lambda \in \operatorname{Core}(v)$, then by Theorem $\mathrm{D}$, there exists $p \in$ $\partial \bar{f}(\mu(T))$ such that $p \cdot \mu(T)=v(T)$ and $\lambda=p \cdot \mu$. It is clear that $p \geq 0$ and $p_{i}=0$ for every $i$ in which $\mu_{i}(T)>v(T)$. Therefore $v(T)=v(T) \sum_{i}^{m} p_{i}$. Now if $v(T)=0$ the result is trivial. If $v(T)>0$ then $\sum_{i}^{m}{ }_{1} p_{i}=1$ and thus Core $(v) \subset$ co $M$.

We consider now a pure exchange economy $E$ in which the commodity space is $\mathfrak{R}_{+}^{m}$. The traders' space is represented by a measure space $(T, \Sigma, \mu)$, where $T$ is the set of traders and $\mu$ is a non-atomic probability measure on $\Sigma$. A coalition is a member of $\Sigma$. An assignment (of commodity bundles to traders) is an integrable function $\boldsymbol{x}: T \rightarrow \mathfrak{R}_{+}^{m}$. There is a fixed initial assignment $\omega$. $(\omega(t)$ represents the initial bundle density of trader $t)$. We assume that $\int_{T} \omega d \mu \gg 0$. Each trader $t \in T$ has a utility function $u_{t}: \mathfrak{R}_{+}^{m} \rightarrow \mathfrak{R}_{+}$.

We first study the case in which all the traders in the economy $E$ have the same utility function $u$ which is continuous, non-decreasing, concave and homogeneous of degree one on $\mathfrak{R}_{+}^{m}$. The Aumann-Shapley-Shubik market game which is associated with the economy $E$ (see Section 30 of Chapter VI in Aumann and Shapley (1974)) in this special case is defined by

$$
\begin{gathered}
v(S)=\sup \left\{\int_{S} u(\boldsymbol{x}(t)) d \mu \mid \boldsymbol{x}\right. \text { is an assignment such that } \\
\left.\int_{S} \boldsymbol{x} d \mu=\int_{S} \omega d \mu\right\} .
\end{gathered}
$$

Proposition 5.1. Assume that every trader in the economy E has the same utility function $u: \mathfrak{R}_{+}^{m} \rightarrow \mathfrak{R}_{+}$which is continuous, non-decreasing, concave, homoge- 
neous of degree one, and satisfies $u(0)=0$. Let $v$ be the market game which is defined in (5.1). Then for every $S \in \Sigma$ we have $v(S)=u\left(\int_{S} \omega d \mu\right)$ and

$$
\operatorname{Core}(v)=\left\{p \cdot \int \omega d \mu \mid p \in \partial u\left(\int_{T} \omega d \mu\right)\right\}
$$

Proof: From the definition of $v$ it is clear that for every $S \in \Sigma$ we have $v(S) \geq$ $u\left(\int_{S} \omega d \mu\right)$. Let $S \in \Sigma$. Since $u$ is concave and homogeneous of degree one, by Jensen's inequality, for every assignment $\boldsymbol{x}$ such that $\int_{S} \boldsymbol{x} d \mu=\int_{S} \omega d \mu$ we have $\int_{S} u(\boldsymbol{x}) d \mu \leq u\left(\int_{S} \omega d \mu\right)$. Therefore $v(S) \leq u\left(\int_{S} \omega d \mu\right)$ and thus $v(S)=$ $u\left(\int_{S} \omega d \mu\right)$. Now (5.2) follows from Theorem A and Theorem D. Q.E.D.

Note that since the function $u$ of Proposition 5.1 is homogeneous of degree one on $\mathfrak{R}_{+}^{m}$, every $p \in \partial u\left(\int_{T} \omega d \mu\right)$ is a vector of competitive prices which corresponds to a transferable utility competitive equilibrium of the economy $E$ (see Section 32 on page 184 of Aumann and Shapley (1974)).

We now apply Theorems A and D to the case when the economy $E$ has a finite number of types.

Two traders in the economy $E$ are of the same type if they have identical initial bundles and identical utility functions. We assume that the number of different types of traders in $E$ is finite and it will be denoted by $n$. For every $1 \leq i \leq n$ we denote by $T_{i}$ the set of traders of type $i$. We assume that $T_{i}$ is measurable and $\mu\left(T_{i}\right)>0$. The utility function of the traders of type $i$ will be denoted by $u_{i}$, and their initial bundle by $\omega_{i}$. We assume that for every $1 \leq i \leq n, u_{i}$ is non-decreasing, concave, and continuous on $\mathfrak{R}_{+}^{m}$.

The Aumann-Shapley-Shubik market game (e.g., Shapley and Shubik (1969) and Aumann and Shapley (1974)) which is associated with the economy $E$ in the case of a finite number of types is

$$
\begin{aligned}
v(S)=\sup \{ & \sum_{i}^{n} \int_{S \cap T_{i}} u_{i}(\boldsymbol{x}(t)) d \mu \mid \boldsymbol{x} \text { is an assignment such that } \\
& \left.\int_{S} \boldsymbol{x} d \mu=\int_{S} \omega d \mu\right\} .
\end{aligned}
$$

Define now a function $f: \mathfrak{R}_{+}^{n} \times \mathfrak{R}_{+}^{m} \rightarrow \mathfrak{R}$ by

$$
f(y, z)=\max \left\{\sum_{i=1}^{n} y_{i} u_{i}\left(x_{i}\right) \mid x_{i} \in \mathfrak{R}_{+}^{m}, \sum_{i=1}^{n} y_{i} x_{i} \leq z\right\} .
$$

Then by Lemma 39.9 of Aumann and Shapley (1974), $f$ is concave, nondecreasing, and homogeneous of degree one on $\mathfrak{R}_{+}^{n} \times \mathfrak{R}_{+}^{m}$.

Proposition 5.2. Let $v$ be the market games which is given by (5.3). Define an $(n+m)$-dimensional vector of non-atomic measures $\xi$ on $\Sigma$ by

$$
\xi(S)=\left(\mu\left(S \cap T_{1}\right), \ldots, \mu\left(S \cap T_{n}\right), \int_{S} \omega d \mu\right) .
$$


Let $f$ be the function which is defined (5.4). Then $v=f \circ \xi$ and

$$
\operatorname{Core}(v)=\{p \cdot \xi \mid p \in \partial f(\xi(T))\} .
$$

Proof: By Lemma 39.16 of Aumann and Shapley (see also Lemma 4.6 in Dubey and Neyman (1981)), for every $S \in \Sigma$ we have $v(S)=f(\xi(S))$. As we noted, $f$ is concave, non-decreasing, and homogeneous of degree one on $\mathfrak{R}_{+}^{n+m}$ (e.g., Lemma 39.9 of Aumann and Shapley (1974)). The concavity of $f$ on $\mathfrak{R}_{+}^{n+m}$ implies that it is continuous on int $\mathfrak{R}_{+}^{n+m}$ and in particular at $\xi(T)$, therefore (5.5) follows from Theorem A and Theorem D and the homogeneity of $f$.

Q.E.D.

\section{References}

1. Aumann RJ, Shapley LS (1974) Values of non-atomic games. Princeton University Press

2. Billera LS, Raanan J (1981) Cores of non-atomic linear production games. Mathematics of Operations Research 6:420-423

3. Dubey P, Neyman A (1984) Payoff in non-atomic economies: an axiomatic approach. Econometrica 52:1129-1145

4. Dvoretsky A, Wald A, Wolfowitz A (1951) Relations among certain ranges of vector measures. Pacific Journal of Mathematics 1:59-74

5. Einy E, Holzman R, Monderer D, Shitovitz B (1996) Core and stable sets of large games arising in economics. Journal of Economic Theory 68:200-211

6. Holmes RB (1975) Geometric functional analysis and its applications. Springer Verlag

7. Kannai Y (1992) The core and balancedness. In: Aumann RJ, Hart S (eds) Handbook of Game Theory with Economic Application, Volume 1, Elsevier Science Publishers, pp. 543590

8. Mertens JF (1980) Values and derivatives. Mathematics of Operations Research 5:523-552

9. Schmeidler D (1972) Cores of exact games I. Journal of Mathematical Analysis and Applications 40:214-225

10. Shapley LS, Shubik M (1969) On market games. Journal of Economic Theory 1:9-25

11. Sion B (1958) On general minimax theorems. Pacific Journal of Mathematics 8:171-176 